\title{
Latitudinal Distribution of the Coronal Bright Points at Solar Minimum and the Rising Phase of Solar Activity Cycle 23
}

\author{
I. Sattarov ${ }^{1}$, A.A. Pevtsov ${ }^{2}$, N.V. Karachek ${ }^{1}$, and A.M. Tillaboev ${ }^{1}$ \\ ${ }^{1}$ Tashkent State Pedagogical University, 103 Usif Xos-Hojib St., Tashkent 700100, Uzbekistan, \\ email: isattar@astin.uzsci.net \\ ${ }^{2}$ National Solar Observatory†, Sunspot, NM 88349, USA \\ email: apevtsov@nso.edu
}

\begin{abstract}
We show that the latitudinal distribution of the coronal bright points (BPs) changes between the solar minimum and the rising phase of solar cycle 23. In 1996, the number of BPs peaks near the disk center. In 1998, the distribution shows bands of increased BPs' number at the ARs' latitudes.
\end{abstract}

\section{Analysis and Results}

We have developed software for automatic identification of the coronal bright points (BPs), and have applied this software to the SoHO/EIT data. Figure 1 shows the examples of BPs identified by our software. Near the solar minimum of cycle 22 (Figure 1a), the distribution of BPs peaks at the disk center. On the rising phase of the solar cycle 23 (Figure 1b) the number of BPs is enhanced at the active regions' (ARs) latitudes. Furthermore, there appears to be a concentration of BPs in two areas situated near the central meridian at $30^{\circ}$ latitudes in both hemispheres. A new AR developed in the northern area a few days later.

The latitudinal distributions of BPs observed on 31-Mar-96 and 3-Feb-98 (not shown) support the visual inference from Figure 1. In 1996, the distribution of BPs peaks near the disk center (see also Figure 2a). The distribution of BPs for 3-Feb-98 shows two maxima at about $27^{\circ} \mathrm{S}$ and $30^{\circ} \mathrm{N}$ latitude. The total number of BPs is larger on 3-Feb-98. This suggests that during the periods of higher sunspot activity there is an additional subset of BPs situated at the ARs' latitudes. Similar double-peaked distributions were observed during other days in February 1998. Figure 2 presents distribution of BPs over several days in 1996 and 1998. During February 1998 (Figure 2b) the active regions' activity was higher in the northern hemisphere. The distribution of BPs is clearly asymmetric with an enhanced maximum in the northern hemisphere.

\section{Conclusions}

(a) The latitudinal distribution in deep solar minimum (1996) peaks at the solar disk center.

(b) The distribution of BPs in quiet Sun is not uniform; there are areas with higher concentration of BPs. Later, a new active region has developed in one of such areas.

(c) Beginning a new sunspot cycle 23, the latitudinal distribution of BPs shows two maxima corresponding to the activity belts.

$\dagger$ Operated by the Association of Universities for Research in Astronomy (AURA, Inc) under cooperative agreement with the National Science Foundation (NSF). 

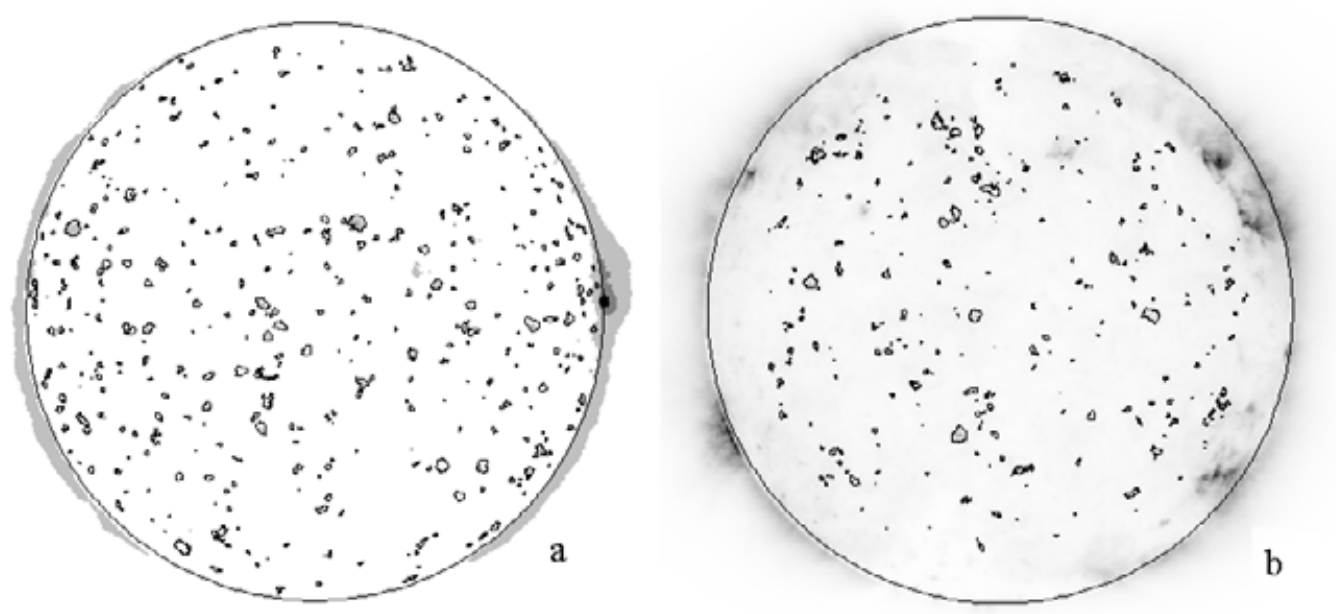

Figure 1. Bright points (contours) identified in $195 \AA$ images on (left) 31-Mar-96 and 3-Feb-1998 (right). On 3-Feb-98, the angular distance for identification was reduced to 0.85 to exclude several active regions near the limb.
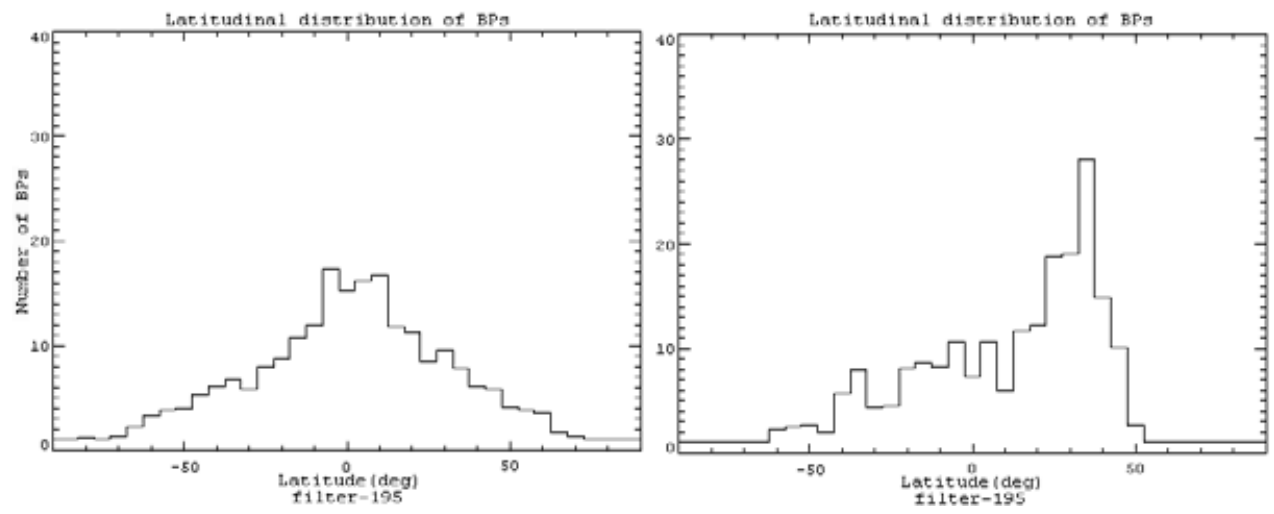

Figure 2. BPs observed in $195 \AA$ from (a) 28-Mar-96 - 30-Apr-96 and (b) 3-5 February 1998.

(d) The total number of BPs in 1998 observations is about $10 \%-20 \%$ (central part - full disk) larger than in 1996 (deep solar activity minimum). The increase comes from the activity belts.

\section{Acknowledgements}

This study was stimulated during the visit of Dr. I. Sattarov to the NSO/SP in a framework of the IAU's Astronomers Exchange Program. 